This item was submitted to Loughborough's Research Repository by the author.

Items in Figshare are protected by copyright, with all rights reserved, unless otherwise indicated.

\title{
Email training significantly reduces email defects
}

PLEASE CITE THE PUBLISHED VERSION

PUBLISHER

(C) Elsevier

LICENCE

CC BY-NC-ND 4.0

REPOSITORY RECORD

Burgess, Anthony K., Thomas Jackson, and Janet Edwards. 2019. "Email Training Significantly Reduces Email Defects". figshare. https://hdl.handle.net/2134/2192. 
This item was submitted to Loughborough's Institutional Repository by the author and is made available under the following Creative Commons Licence conditions.

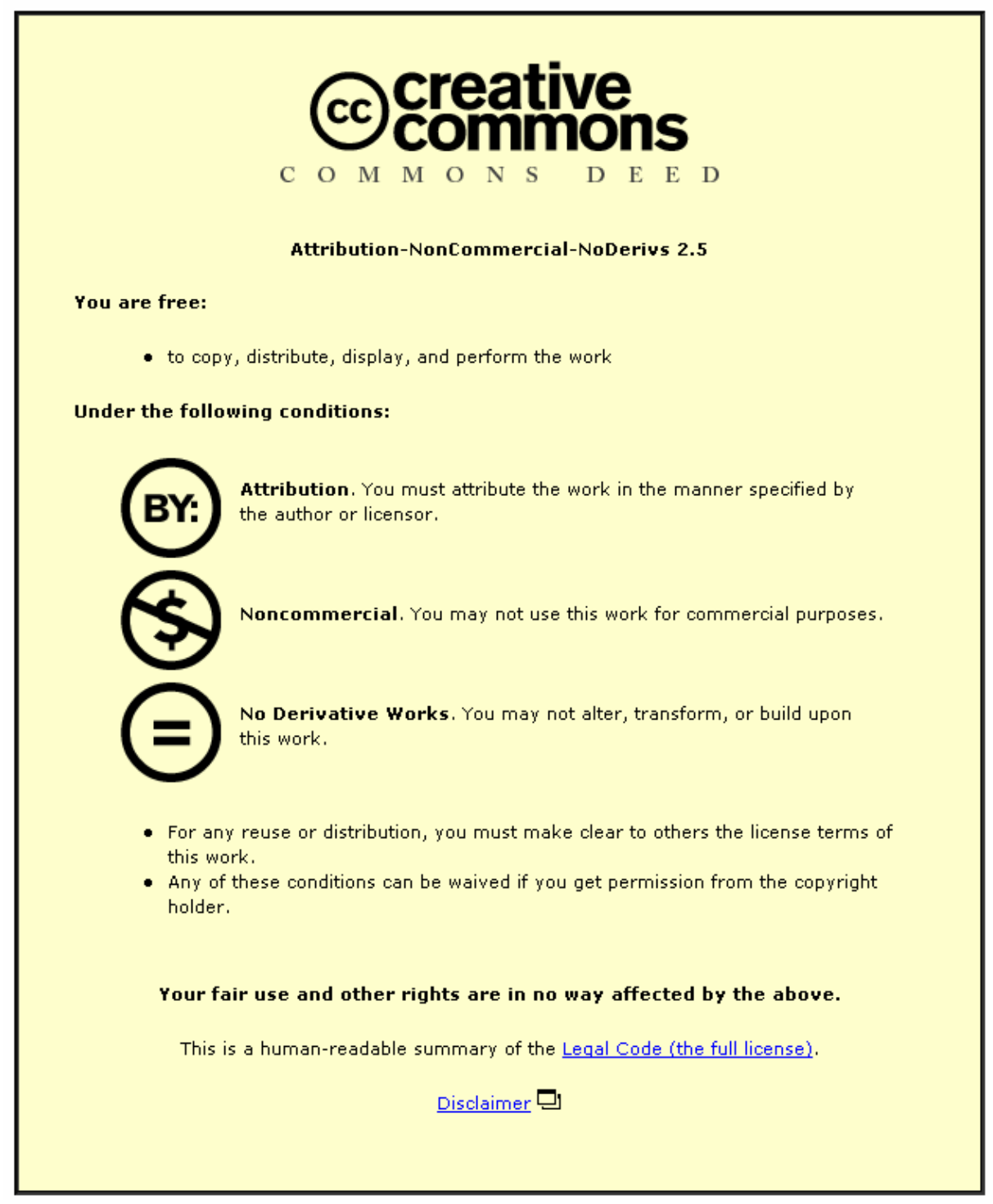

For the full text of this licence, please go to: http://creativecommons.org/licenses/by-nc-nd/2.5/ 


\title{
Email Training Significantly Reduces Email Defects
}

\author{
Anthony Burgess ${ }^{*}$, Thomas Jackson and Janet Edwards \\ Computer Science Department, Loughborough University, Leicestershire, UK
}

\begin{abstract}
Organisations are now becoming aware of the problems associated with email use and are keen to reduce these defects. These email defects relate to the ineffective way that email is used within organisations, and are not only limited to the volume of email that is sent and received, but also the quality of the email content. Email defects lead to inefficiencies within the workplace as employees spend more time dealing with email rather than doing other aspects of their job. This paper firstly examines how email is used within a large organisation and highlights the defects associated with email. The initial results show that these defects affect some groups of employees more than others. The paper also reports on the effectiveness of email training in reducing the defects associated with email use. The results show that some of these defects are related and that training can significantly reduce some of the email defects and improve the way people write emails.
\end{abstract}

Key Words: Email, Electronic communication, Employee training, Employee productivity.

*Corresponding author. Computer Science Department, Loughborough University, Loughborough, Leicestershire LE11 3TU, UK. Tel.: +44 (0)1509 228230; Fax: +44 (0)1509 211586.

Email address: a.k.burgess@lboro.ac.uk (A. Burgess). 


\section{INTRODUCTION}

As new communication methods are introduced into organisations it is important to understand how and why managers use the new media (Markus, 1994a), as it is not the media per se that determines communication patterns, but rather the social processes surrounding media use (DeSanctis and Poole, 1994). Email is beginning to dominate the day-to-day communications within business organisations (Murphy et al, 1997). The International Data Corporation (IDC) predicts that 35 billion emails will be sent every day by 2005. There is also an increasing variety of information types carried by email, resulting in employees spending a greater deal of time interacting with email applications, which have become their primary "electronic habitat” (Ducheneaut and Bellotti, 2001). The habitat is increasingly being used for tasks for which it was not designed, such as task management and personal archiving, which increases the chance of employees becoming overwhelmed by email (Whittaker and Sidner, 1996). By understanding communication methods, the mediums can be optimised to enhance performance within organisations. We still have much to learn about interactions among computer-mediated communication technologies, new organisational forms, and changes in work and communication (Daft and Lewin, 1993), but the ability to monitor email now provides organisations with the opportunity to improve communication practices.

As communication pervades nearly everything we do, even small improvements in the effectiveness and cost of our communication processes can have significant benefits. However, the majority of employees are not taught how to become effective users of email (Nantz and Drexel, 1995), which maybe because such skills are taken for granted. Email education within organisations tends to focus on the hardware and 
software issues without regard for the requisite communication skills (Nantz and Drexel, 1995). Even the most educated of employees can lack the basic skills for expressing themselves effectively (Davenport, 1997).

The paper builds on published work, by Solingen et al (1998) and Jackson et al (1999), identifying email productivity and efficiency costs and generating questions on how to enhance performance throughout an organisation using electronic networks. The research within this paper involved employees identifying problems with email communication within the workplace and the impact of them on the employee. To reduce the identified email defects, specific training was developed and administered to a pilot cohort of employees at a large UK Plc. The research study identifies problems with email communication and demonstrates how email training can reduce email defects within the workplace to enhance email communication.

\section{IDENTIFIED PROBLEMS WITH EMAIL COMMUNICATION}

Organisations are now becoming increasingly aware of the problems that are associated with email use within the workplace. These problems, referred to as email defects, are not only limited to the volume of emails that are sent and received each day, but also to the quality of the actual emails themselves.

\subsection{Information Deficiency}

Research has shown that more than $65 \%$ of all email messages fail to give the recipients enough information to act upon, and ambiguous poorly written email can lead to misunderstandings that can cause tension within the workplace (Frazee, 1996). This may lead to incorrect instructions being carried out, which can have serious 
implications for an organisation if the wrong information is used to make decisions. Messages can appear to be ambiguous if the sender fails to understand the context into which their messages are being received (Kimble and Abu Bakar, 2001).

\subsection{Poorly Targeted Emails}

In their study within a large international organisation Kimble et al, (1998) found that some managers were overloaded with emails because of the inappropriate use of the cc (Carbon Copy) function. Employees may often copy emails to their superiors simply to "cover their own back". The cc function is also often used to send emails that are for information only and do not require action, however the recipient does not know this and has to spend time processing the message assessing if action is required upon their part.

\subsection{Media Section}

Markus (1994b) found that employees would often choose email in place of other forms of communication in circumstances where they wished to avoid unwanted social interaction, such as when communicating with someone intimidating or someone they do not like. The communication medium must be appropriate for the message that is to be transmitted. Valacich, et al (1993) found that email was more effective than face-to-face communication when used by groups for a task of low ambiguity. This suggests that face-to-face communication is better suited to tasks with a high level of ambiguity where groups are likely to have to make a decision, and email is better suited to tasks of low ambiguity. 


\subsection{Interruption}

Email can be a distraction for employees, especially if it interrupts them from more important work. Research undertaken by Jackson et al (2001a) found that the amount of time it takes employees to recover from an email interrupt, and to return to their work at the same rate at which they left it, was found to be on average 64 seconds. Jackson et al (2001a) suggest that it is possible for employees to become more efficient if they change the duration in which their email application checks for new email.

\subsection{Processing and Filing of Email}

The growth in the volume of email can make it difficult for employees to prioritise and manage their email messages. Whittaker and Sidner (1996) discovered that users of email found it difficult to file away emails that were read, meaning that large numbers of emails tended to stay in the users inbox. This may be because users may find it difficult to categorise their email when archiving, especially if they wish to be able to retrieve them easily at a later date. Users often have trouble retrieving messages for later use or in remembering to reply or to act upon a particular message because current email systems are designed around the assumption that messages are informational and are read upon arrival, and that important messages are filed (Yiu et al, 1997). The poor use of the subject line can magnify this issue. If the subject line of an email is empty or inappropriate then it is harder for the recipient to prioritise and process the message.

The above research has shown some of the known problems with email communication within the workplace. However, the majority of the research does not 
quantify the impact of email defects on employees and how these can be reduced to enhance email communication between employees.

\section{METHODOLOGY}

The research for this paper was undertaken at a large UK Plc, which is a diversified technology company with manufacturing, sales and marketing operations. The research involved contacting all email users (2,850 employees) within the organisation in both the UK and Ireland. Although only one organisation was surveyed the results of this study are expected to be similar to that of other organisations of a similar size and structure.

\subsection{Identifying Email Defects}

The study at the large UK Plc was conducted with the aim of creating an overall representation of the current state of email defects within the organisation. A questionnaire was developed to capture employees’ views on how email was used within the organisation. As the study was aimed at the whole organisation a questionnaire was constructed as it would have been impractical due to time restrictions to undertake a one-to-one interview approach. It would also have been impractical to monitor such a large number of employees to get the information required. The questionnaire developed by the authors was securely hosted on the Internet, which made capturing the data easier than if a paper based questionnaire had been used. Each response to the questionnaire was automatically stored in a database, with each column representing each question, and each row representing each submitted response. The questionnaire was hosted at Loughborough University, not 
at the organisation, to ensure that the data would not be manipulated prior to analysis and to give the employee's added security that their individual responses would not be disclosed to the management at the organisation.

The questionnaire asked employees to specify how many emails they received on average each day and what proportions of these were irrelevant or for information purposes only. Employees also answered questions that related to the defects associated with email and to what extent the employees were affected by the defects. The employees were asked to specify their age, job grade, department, and how long they had worked within the organisation. This made it possible to compare the responses to employees with different demographic characteristics.

\subsection{Reducing the effect of the identified email defects through Training}

With the identification of the email defects in phase-one of the research, the second phase of the research was to reduce the identified email defects through constructing and deploying specific training to a small group of employees. There were 20 sender and recipient pairs that participated in the experiment. The recipients were asked to mark up to 20 emails that they received from the sender before and after the sender had received training on the best practice of email use. The recipients would mark each email against a set of criteria, giving a score depending on how well the email met each criterion. The scores given were on a 5-point scale, with a score of 1 meaning that the email met the criterion and a score of 5 meaning that it did not meet the criterion at all. The evaluation criteria related to the email defects identified within the initial study. The senders were shown a list of the criteria by which they would be evaluated, although they did not know who would be evaluating their emails. The 
scores both before and after the training were averaged for each sender / recipient pair. The average of all 20 pairs was calculated to determine the overall effect of the training for each of the criterion. The chosen pairs for the experiment were based on high volume email senders with a recipient that was likely to receive a high number of sender's emails during the two-week monitoring process (one week before and after training). Due to the time constraints of two-weeks, only high volume senders were chosen to maximize the probability that 20 emails would be received by the recipient of the pair.

\subsection{Training Employees on the Best Practice of Email use}

Both sender and recipient groups received training on the best practice of email use for this study. Both training sessions were seminar based and teleconference facilities used for participants in other locations. There were two dates for both training sessions as not all participants were able to make the same day. This meant that there were around 4 -10 participants in each training session. Each session was scheduled for one hour.

The recipients training focused mainly on the assessment criteria. Recipients were given guidance as to how to complete the evaluation sheet, and an explanation of the scale that would be used to mark each of the criteria. The recipients were given a presentation that highlighted the problems of email use within the company.

The sender training went into more detail to explain the email defects. This was also more interactive than the recipient training as the senders were shown examples of poor emails and were asked to pick out the defects, whereas the recipients were 
simply told where the defects were. The senders were given training on how to better manage their email by the use of folders and archiving. The senders were shown the evaluation sheet so that they knew what they would be marked against. The training for the senders was more comprehensive than that of the recipients because it was the senders that were being marked, whereas the recipients just needed to be aware of the email defects and be able to complete the evaluation sheet. The sender training also followed a structured presentation, although this was more interactive that the recipient training.

\section{QUESTIONNAIRE RESULTS - EMAIL DEFECTS}

The company has 2850 copies of Lotus notes, which is the email application used within the UK and Ireland, and all users of the application were asked to fill in the questionnaire (2850 employees). Some 875 responses were received giving a response rate of 31\%. The results from this study concur with section two of this paper on the known problems with email. However the questionnaire results also provide quantitative data that has enabled further analysis to be undertaken to determine the underlying defects of email and how they can be reduced.

The results showed that the average number of emails received each day by an employee was 23, which is lower than a similar study undertaken by Mackay (1989) (average of 39 emails received per day) and similar to a study by Frazee (1996) (average of 15 emails received per day). These subjective studies are not too dissimilar to the actual monitoring of email accounts at the Danwood Group by Jackson et. al. (2003), who found on average 15 emails were received per day. 


\subsection{Information Deficiency}

Employees were asked if the subject line of an email contained sufficient information for them to be able to assess the importance of the email. Only $27 \%$ gave a positive response to this, $45 \%$ gave a negative response, and $28 \%$ were neutral. Poor use of the subject line can make it difficult for the recipients to prioritise incoming messages and as a result they may fail to action some emails on time.

\subsection{Poorly Targeted Emails}

On average $13 \%$ of the emails employees received were irrelevant or untargeted. This would indicate that employees spend a considerable amount of time reading emails that are not directly relevant to their job. The majority of comments that were made in the optional comment box were related to the way that employees receive untargeted email. The employees complained about the overuse of the 'reply-to-all' function, or the inaccuracy of mailing lists. The consequence of the 'reply-to-all' is that it increases network traffic and increases the time employees spend reading irrelevant or untargeted emails. On average $41 \%$ of the emails received are for information purposes, and on average employees believe that they are copied in unnecessarily on $16 \%$ of the emails they receive.

\subsection{Media Selection}

Other comments made in the optional comment box were related to email being increasingly used instead of other forms of communication. This was enforced by some $56 \%$ of respondents agreeing that email is too often used when face-to-face communication or the phone should be used instead. This would have a massive 
impact on the amount of unnecessary email traffic that was generated within an organisation. Time would also be wasted writing and reading emails that contain information that would have been more effectively communicated by another medium.

\subsection{Email Interruptions}

Email was found to be a distraction by $49 \%$ of employees, who said that it often distracts them from other aspects of their work. Although there was found to be no correlation between whether employees thought email was a distraction and whether they had a pop up notification when they received new email as suggest in earlier research by Jackson et. al. (2001a). If employees are distracted by email on a frequent basis this will have a detrimental effect on their work (Jackson et. al. 2003).

\subsection{Processing and Filing of Email}

When asked if the emails they receive are easy to read $45 \%$ of respondents gave a positive answer, while 10\% gave a negative response and 45\% were neutral. Emails that are difficult to read take longer to understand and the message may be misinterpreted if it is ambiguous. Only $46 \%$ of employees said that the actionable emails they receive state what action is expected of them. On average $8 \%$ of the emails received each day are either difficult to understand, or employees found the purpose of the message unclear. If employees do not understand a particular message then this can lead to a delayed action. Emails of this nature can also cause the recipient to misinterpret the message, which can have disastrous implications for the organisation. It is also important that emails are to the point. If the message is complex then it maybe more appropriate to speak in person, not only to save on the 
time writing the email, but also to reduce the risk of the message being misinterpreted. Just over one third of employees (37\%) said that when they receive an actionable email it does say when action is required. This would indicate that some actions might not be completed when expected because the recipient was unaware of any deadline.

\section{EMAIL DEFECTS ARE RELATED TO JOB GRADES, AGE AND DURATION OF EMPLOYMENT}

The research study has highlighted that the extent of the email defects are directly related to job grade, age and duration of employment.

\subsection{Job Grades}

Employees were asked to indicate their job grade on the scale shown in Table 1. This enabled comparisons to be made between how employees at different levels within the organisation viewed email use.

\section{Insert Table 1 here}

From Table 1 it can be seen that employees with higher job grades received on average more email than those with lower job grades. This could be partly due to employees copying in their managers on emails just to "cover their own back".

It was found that respondents with a higher job grade tended to be more critical of the emails they received compared to those on lower job grades. This may be influenced 
by the fact that a higher proportion of respondents with a higher job grade answered positively when asked if they received irrelevant or untargeted email, or felt that they were copied in unnecessarily than respondents from lower job grades.

Respondents from higher job grades thought email was used too much in place of other forms of communication compared to lower job grades. This indicates those on lower job grades wrote emails where the message may be better suited to another form of communication. Employees on higher job grades may be more concerned about the overuse of email compared to their subordinates because they receive on average more email, as shown in Table 1. Those on higher job grades gave a stronger indication that email often distracts them from more important work. This may also be due to the volume of emails received by those on higher job grades.

The results from the questionnaire indicate that those with higher job grades are more critical of email use within the organisation and that they can relate to the problems that are associated with email use, compared to those respondents with lower job grades.

\subsection{Age}

Comparisons can also be drawn between how respondents from the different age groups view email use within the organisation. The age range of the respondents in this study is shown in Table 2.

\section{Insert Table 2 here}


Older aged employees had a stronger response (60\% for those between 25 and 44 ) to whether email is used too often instead of other forms of communication compared to those in the Under 25 category (42\%). This indicates that younger employees find the balance between email and other communication methods to be more acceptable.

When employees were asked to evaluate how easy the emails they received are to read, the Under 25's gave a positive response of 53\% compared to other age categories. This shows that younger employees tend to be less critical of the emails they receive when compared to older employees.

Just over half of the 35 to 44 year old employees (53\%) indicated that they received unnecessary or irrelevant emails. The Under 25's and Over 55's are significantly less affected (34\%, 26\% respectively). The same trend is true when employees were asked if email sometimes distracts them from other work. The fact that younger employees are less concerned about the distracting nature of email and less troubled about receiving irrelevant or untargeted email relates to the younger employees not being aware or affected in the same way by the problems associated with email. The over 55 year olds may share a similar view on these issues, not because they are unaffected by the problems of email, but because they accept these problems and no longer consider them an issue to complain about.

\subsection{Duration of Employment}

The study has shown that employees had different views about email use depending on how long employees had worked within the organisation. The views of an 
employee who has been employed within the organisation for over 15 years were found to be different from those of an employee who has recently joined the organisation. Employees that had been employed for more than five years on average received slightly more emails than employees who had been with the organisation for less than five years.

Individuals that have been employed for longer tend to be more critical of the emails they received in terms of whether they are easy to read, to the point, and tell them what is expected of them compared to those who had been with the organisation for only a few years.

The employees who were more critical of the emails they received were employed for longer, and also had stronger opinions on whether email is used too much in place of other forms of communication. Also respondents who had worked within the organisation for longer had stronger views when asked if they thought they received irrelevant or untargeted email, or if they felt they were copied in unnecessarily on email.

This shows that the employees that have been employed for a longer period of time are more critical of the emails they receive because they consider them to be irrelevant and untargeted email and some of the emails better suited to another form of communication.

\section{THE EFFECT OF TRAINING ON REDUCING EMAIL DEFECTS}


From the data obtained from the second phase of the research it was possible to determine which email defects were most receptive to email training. As part of this research the recipients have evaluated the emails sent to them by their paired sender. These values were then averaged and combined for all twenty sender and recipient pairs. The overall effect of the training is shown in Table 3, where the averages for both before and after training have been shown.

\section{Insert Table 3 here}

Table 3 shows that there has been an improvement in the quality of emails received by the recipients in this experiment as a result of email training for the senders. The numbers shown in Table 3 indicate the extent of the shift along the 5-point scale as a result of the training, except for the last criterion in the table that is in seconds. It is inappropriate to apply percentage changes to indicate the success of the training because percentages can exaggerate the real change depending on where on the scale the change occurs. For example a change from 5 to 4 and 2 to 1 both show a change of 1 , but show percentage changes of $20 \%$ and $50 \%$ respectively.

\subsection{The Significance of the Training}

To determine the significance of the effect of training the t-test statistic was calculated for each of the criterion that the emails were marked against, using the before and after values of the 20 sender and recipient pairs. The t-test statistic for each of the criterion can be seen in Table 4, the values are based on a paired two-tailed t-test.

\section{Insert Table 4 here}


The t-test values in Table 4 show that the training has been significantly successful at the 95\% level at improving an employee’s ability to write emails that are easy to read and that are straight to the point. The results also show that the training has been significant at the 99\% level at improving the way that an employee uses the subject line to convey information about the content and the urgency of an email.

These results in row two and three of Table 4 show the email training had a significant impact on improving the senders' ability to write clearer emails that are straight to the point. This is important because emails that are more direct take less time to read and understand. Emails that are easy to read are less likely to be misinterpreted, so there is less chance of incorrect actions being carried out. Just under half (45\%) of respondents from phase one of the study indicated that the email they receive is easy to read. After training there was an increase in the number of easy to read emails received and is expected that through the deployment of email training throughout the whole of the organisation this figure is likely to increase.

The training has had a highly significant impact on improving the senders' ability to make better use of the subject line. As a result of the training the senders were writing subject lines that better enabled the recipient to know what the message is about and to judge the importance of the message. If a message has an informative subject line then it is easier for the recipient to process the email (Jackson et. al. 2001b). This is important for employees that receive large numbers of email and find it difficult to process their inboxes. Only $27 \%$ of respondents from the initial study indicated that 
the subject line contains sufficient information for them to assess the importance of the email.

\section{THE RELATIONSHIP BETWEEN EMAIL DEFECTS}

As part of this research it was important to assess the strength of the relationship between the different criteria. Through understanding the relationships it is then possible to give further meaning to the significance of the email training. For instance it was found that if an email was straight to the point then it was also likely to be easy to read. The relationships were calculated using Pearson's correlation coefficient (r) to determine if there was any significance in the relationships between how the recipients marked the emails against one criterion compared to another criterion. This was done for emails evaluated before and after the senders had received training.

This analysis does not show if there has been any improvement in any of the criteria, it only shows if there is a relationship between how two of the criteria were marked. A significant correlation would show that one criterion is marked in the same way as another, but it will not show if the training has lead to an improvement or not.

It was found that there was positive correlation at the $95 \%$ level for the relationships, shown in Table 5, both before and after the senders had received training.

\section{Insert Table 5 here}


The results in Table 5 shows that there is a relationship between whether an email is easy to read, whether it is to the point and whether email was the most appropriate medium to use. It can be said with $99 \%$ confidence that there is a correlation between an email being easy to read and being to the point. The data shows that messages that would have been better suited to another form of communication are likely to be difficult to read and not to the point. An email that is easy to read is likely to be straight to the point; similarly an email that is difficult to read is likely not to be to the point. There was also a significant relationship between whether an email is straight to the point and whether it is relevant to the recipient. Messages that are relevant to the recipient are likely to be straight to the point, whereas long rolling emails are likely to be irrelevant to the recipient.

The criteria that are associated with actionable emails are related. If an actionable email states what action is required then is likely that the email will also state by when the action is required. Although the training did not show a significant improvement in this area, it is useful for training purposes that employees who write actionable emails also specify when the action is required by.

Similarly the criteria associated with the use of the subject line are related. If a recipient is able to access the importance of an email from the subject line they are also likely to know what the message is about. Effective use of the subject line is vital for recipients who often prioritise and manage incoming email based on the subject line. 


\section{CONCLUSION}

The results from this study highlight the many problems that are often associated with email use within organisations. In particular, this research study showed that the extents of the email defects are directly related to job grade, age and duration of employment. The more senior employees within the studied organisation suffered the most from the email defects compared to their more junior colleagues. The effect of an email defect becomes greater the more email is received and in this study the more senior employees received more email than that of their junior counterparts.

Receiving email on an exponential rate becomes harder to manage and prioritise, so the effect of poorly worded emails and inadequate subject lines compounds the situation through additional time spent dealing with emails with defects.

The correlation analysis has shown that there is a statistically significant relationship between some of the evaluation criteria which were used in the training experiment. These relationships can then aid in the understanding of how an improvement in one area can lead to an improvement in another, which can enable detailed and specific email training to be prescribed to specific employees.

Training employees on the best practices of email use can help to reduce email defects within an organisation. The t-test analysis shows that 4 out of the 9 criteria showed significant improvements after the senders had received training. The four significant improvements were: 
- (99\% significant) better use of the subject line which makes it easier to prioritise an email and also to assess the content

- (95\% significant) better written emails which were easier to read and to the point.

The research has been unable to determine who within an organisation requires the appropriate training, as the research concentrated mainly on the recipients and not the senders. However, it has shown that email training can help reduce the number of email defects within an organisation. 


\section{REFERENCES}

Daft R.L., Lewin A.Y., "Where Are the Theories for the 'New' Organizational Forms?”, Organizational Science, Vol.4, No.4, pp.I - IV, 1993.

Davenport, T. H., (1997) Information Ecology, Oxford University Press, New York

DeSanctis G., Poole M.S., "Capturing the Complexity in Advanced Technology Use: Adaptive Structuration Theory”, Organizational Science, Vol.5, No.2, pp.121 - 147, 1994.

Ducheneaut, N., \& Bellotti, V. (2001) Email as habitat: an exploration of embedded PIMM Interactions, 8 (5), ACM Press. 30-38.

Flash Commerce (2000) Daily Email Output to Reach 35 billion by 2005 http://flashcommerce.com/articles/00/10/11/193202920.html

Frazee, V.(1996). Is e-mail doing more harm than good? Personnel Journal, 23.

Jackson T.W., Dawson R., Wilson D., "Improving the Communications Process: The costs and effectiveness of email compared with traditional media”, INSPIRE Training and Teaching for the understanding of Software Quality”, No.4, pp.167 178, September 1999.

Jackson, T. W., Dawson, R. J. and Wilson, D., 'The Cost of Email Interruption', The Journal of Systems and Information Technology, 5(1), 2001a, pp81-92, ISSN 13287265

Jackson, T.W., Dawson, R.J. and Wilson, D., “The Cost of Email Interruption”, The Journal of Systems and Information Technology, 5(1) , 2001b, pp. 81-92, ISSN 13287265 .

Jackson, T.W., Dawson, R.J. and Wilson, D., "Understanding Email Interaction Increases Organizational Productivity', Communications of the ACM ,46(8), August 2003, pp. 80-84, ISSN: 0001-0782 .

Kimble, C., \& Abu Bakar, J. (2001). Information Overload and Electronic Mail: Tagging Message Content - A Partial Solution? Proceedings of the $6^{\text {th }}$ UKAIS Conference, University of Portsmouth, Zeus Press, April 2001, pp 371-380

Kimble, C., Hildreth, P. and Grimshaw, D. (1998) The role of contextual clues in the creation of Information Overload, Matching Technology with Organisational Needs, Proceedings of the 3rd UKAIS Conference, Lincoln, UK. 405-412.

Markus L. .M., (1994a) "Electronic Mail as the Medium of Managerial Choice”, Organizational Science, Vol.5, No.4, pp.502 - 525, November 1994.

Markus, L. M., (1994b) 'Finding a Happy Medium: Explaining the Negative Effects of Electronic Communication on Social Life at Work, ACM Transactions on Information Systems, Vol. 12, No. 2, April 1994, Pages 119-149 
Murphy, H. A., Hildebradt, H. W., and Thomas, J. P. (1997) Effective Business Communications $\left(7^{\text {th }}\right.$ ed.). New York: McGraw-Hill.

Nantz, K. S., \& Drexel, C. L. (1995) Incorporating electronic mail into the business communication course. Business Communication Quarterly, 58, 45-51

Solingen R., Berghout E., Latum F., "Interrupts: Just a Minute Never Is”, IEEE Software, Vol.15, No.5, pp.97 - 103, September/October 1998.

Valacich, J. S., Paranka, D., George, J. F., and Nunamaker, Jr., J.F. (1993).

Communication Concurrency and The New Media. Communication Research, 20 (2) 249-276.

Whittaker, S. and Sidner, C. "Email Overload: Exploring Personal Information Management of Email. CHI'96 Conference Proceedings, 1996: 276-283

Yiu, K. S., Baecker, R., Silver, N., and Long, B. A Time- Based Interface for Electronic Mail and Task Management. Proceedings of HCI International '1997, Vol $2,19-22$ 
Table 1

\begin{tabular}{lc}
\hline Job Grade (JG) & Average Number of Emails \\
\hline & \\
Upto JG 8 & 22 \\
JG 9 - 10 & 23 \\
JG 11 - 13 & 33 \\
JG 14 + & 44 \\
All Employees & $\mathbf{2 3}$ \\
\hline
\end{tabular}

Table 1 - Average number of emails received by job grade 
Table 2

\begin{tabular}{lc}
\hline Age Range & Proportion of Total Respondents \\
\hline & \\
Under 25 & $8 \%$ \\
$25-34$ & $31 \%$ \\
$35-44$ & $30 \%$ \\
$45-54$ & $25 \%$ \\
Over 55 & $6 \%$ \\
\hline Table 2. Age of Questionnaire Respondents (\%)
\end{tabular}

Table 2. Age of Questionnaire Respondents (\%) 
Table 3

\begin{tabular}{|c|c|c|c|}
\hline Criterion & $\begin{array}{l}\text { Before } \\
\text { Training }\end{array}$ & $\begin{array}{l}\text { After } \\
\text { Training }\end{array}$ & Difference \\
\hline $\begin{array}{l}\text { The message would have been better suited to a } \\
\text { telephone call, or another medium. }\end{array}$ & 1.49 & 1.40 & -0.10 \\
\hline The email is easy to read & 1.63 & 1.39 & -0.24 \\
\hline The email is straight to the point & 1.58 & 1.36 & -0.22 \\
\hline The email is totally irrelevant to me & 1.69 & 1.50 & -0.19 \\
\hline \multicolumn{4}{|l|}{ If it is an actionable email: } \\
\hline It tells me what is expected of me & 1.80 & 1.73 & -0.13 \\
\hline It states when action is required & 2.61 & 2.12 & -0.48 \\
\hline \multicolumn{4}{|l|}{ The subject line contains sufficient detail for: } \\
\hline Me to assess the importance of the message & 2.89 & 2.19 & -0.70 \\
\hline Me to understand what the message is about & 2.17 & 1.49 & -0.65 \\
\hline $\begin{array}{l}\text { Approx how long did it take to read and understand } \\
\text { this message? (Seconds) }\end{array}$ & 76.21 & 65.67 & -10.54 \\
\hline
\end{tabular}

Table 3: The overall mean effect of training on how emails are evaluated 


\section{Table 4}

\begin{tabular}{ll}
\hline & $\begin{array}{l}\text { Significance } \\
\text { from (2 tailed) } \\
\text { t test }\end{array}$ \\
Criterion & 0.438 \\
The suitability of email as the communication medium & 0.022 \\
The email is easy to read & 0.023 \\
The email is straight to the point & 0.138 \\
The relevance of the message to me & \\
If it is an actionable email: & 0.623 \\
$\quad$ It tells me what is expected of me & 0.320 \\
It states when action is required & \\
The subject line contains sufficient detail for: & 0.003 \\
$\quad$ Me to assess the importance of the email & 0.005 \\
$\quad$ Me to know what the message is about & 0.285 \\
Approx how long did it take to read and understand this message?
\end{tabular}

Table 4: t-test statistic comparing the difference between before and after training for each criterion. 
Table 5

\begin{tabular}{lll}
\hline Significant Relationships Between Criteria & $\begin{array}{l}\text { Before } \\
\text { Training } \\
\text { (r) }\end{array}$ & $\begin{array}{l}\text { After } \\
\text { Training } \\
\text { (r) }\end{array}$ \\
\hline $\begin{array}{l}\text { The message would have been better suited to a telephone call, or another } \\
\text { medium AND the email is easy to read }\end{array}$ & $0.646^{*}$ & 0.533 \\
$\begin{array}{l}\text { The message would have been better suited to a telephone call, or another } \\
\text { medium AND the email is straight to the point }\end{array}$ & $0.72^{*}$ & $0.61^{*}$ \\
$\begin{array}{l}\text { The email is easy to read AND the email is straight to the point } \\
\text { The email is straight to the point AND the email is totally irrelevant to me }\end{array}$ & $0.788^{*}$ & $0.7^{*}$ \\
$\begin{array}{l}\text { If it is an actionable email it tells me what is expected AND by when action } \\
\text { is required }\end{array}$ & 0.473 & $0.664^{*}$ \\
$\begin{array}{l}\text { The subject line contains enough information for me to be able to assess the } \\
\text { importance of the message AND for me to know what the message is about }\end{array}$ & $0.815^{*}$ & 0.492 \\
\hline
\end{tabular}

Table 5: Significant relationships between criteria both before and after training

* significant at $99 \%$ level 
Anthony Burgess is a Research Student at Loughborough University. He has a BSc (Hons) degree from Aston University and an MSc degree from Loughborough University. His current research interests include exploring how electronic communication is used within organisations, with a focus on reducing the many problems associated with it.

Thomas Jackson is a Lecturer in Computer Science at Loughborough University. His research areas include Electronic Communication, Electronic Knowledge Management, and Cost Effectiveness of IT Systems. He is currently conducting research into Just in Time Knowledge and using Electronic Messaging to capture Knowledge. Tom has a BSc honours and a PhD from Loughborough University and his on the international review board for the Software Quality Management Conference, Empirical Assessment of Software Engineering Conference, Information Resources Management Conference and the Journal of Electronic Commerce. Tom is currently supervising six PhD students in the Knowledge Management area and they mainly funded by industry, namely, Rolls-Royce, The Danwood Group, AstraZeneca and $3 \mathrm{M}$.

Janet Edwards is a lecturer in Computer Science at Loughborough University. She has a Btech (Hons) degree and an MSc degree from Loughborough University. Her current research interests include CSCW and how students can use such tools in group working. 